\title{
Optimal interventional treatment in a patient with occlusion of the brachiocephalic trunk and left subclavian artery with "double" steal syndrome
}

\author{
Paweł Latacz ${ }^{1}$, Marian Simka², Marek Kazibudzki², Tomasz Ludyga ${ }^{2}$, Piotr Janas², Tomasz Mrowiecki ${ }^{1}$ \\ ${ }^{1}$ Department of Vascular Surgery, University Hospital, Krakow, Poland \\ ${ }^{2}$ Department of Vascular Surgery, EuroMedic, Katowice, Poland
}

Postep Kardiol Inter 2015; 11, 4 (42): 341-343

DOI: $10.5114 /$ pwki.2015.55609

\section{Introduction}

Subclavian steal syndrome, which comprises reversal of flow in the ipsilateral vertebral artery with stenosis or occlusion of the subclavian artery (SA) or brachiocephalic trunk (BCT), was first described by Contorni in 1960 [1]. This clinical syndrome manifests with the signs and symptoms of vertebrobasilar insufficiency, but can also be asymptomatic.

This paper describes a step-by-step technique of complex endovascular treatment for a double steal syndrome caused by occlusions of the BCT and the left SA, associated with stenosis of the left internal carotid artery (ICA) and reversed flow in the left external carotid artery (ECA).

\section{Case report}

A 64-year-old man with a 3-month history of non-specific neurological symptoms, such as vertigo and syncope, was admitted to our hospital. He presented with cardiovascular risk factors: grade 3 hypertension and hypercholesterolemia, and also with bilateral stenoses of the common iliac arteries. Doppler sonography revealed occlusions of the BCT and of the left SA, and stenosis of the left ICA caused by a long atherosclerotic plaque, with increased systolic and diastolic flow velocities to $350 \mathrm{~cm} / \mathrm{s}$ and $120 \mathrm{~cm} / \mathrm{s}$, respectively. In the past, in another medical centre, he underwent an unsuccessful attempt of endovascular revascularization of the left SA.

After a thorough diagnostic evaluation, including assessment of the patient's neurological status and the above-mentioned sonographic examination, we decided to perform catheter angiography of the arteries supplying the brain, in order to obtain more information about cerebral hemodynamics. This examination revealed occlusion of the BCT localized about $1 \mathrm{~cm}$ from the aorta, occlusion of the left SA localized about $2 \mathrm{~cm}$ from the aorta, and also an $80-90 \%$ stenosis of the left ICA (Figures 1 A-C). Morphology of these lesions was typical of atherosclerosis and not of fibromuscular dysplasia or Takayasu disease. Moreover, a detailed analysis of angiographic pictures revealed the presence of a double steal syndrome. First, there was reversed flow via collateral from the left ECA to the left SA, and then to the left vertebral artery (VA) and the basilar artery. This collateral network constituted the main blood supply to the posterior part of the brain, since there was no inflow of injected contrast from the left carotid artery through the Willis circle to the basilar and cerebellar arteries (Figures $1 \mathrm{D}, \mathrm{E}$ ).

Taking into account this unique hemodynamic pattern, and also the general condition of the patient, we decided to reconstruct the arteries using endovascular technique in two stages. Also, as the first procedure we decided to improve the inflow to the posterior part of the brain, reconstructing the left SA and restoring the proper flow in the left VA. An additional benefit of such a therapeutic scenario would be the possibility of adequate management of arterial hypertension (the patient presented with a history of severe arterial hypertension, yet because of bilateral occlusions of the SAs it was impossible to measure his arterial pressure non-invasively; arterial pressure measured intraoperatively in the aorta was 240/140 mm Hg). Reconstruction of stenosed ICA and of occluded BCT was planned as the second step. In our opinion, alternative therapeutic scenarios were associated with a risk of intraprocedural stroke in the posterior brain territory or other severe complications. For example, stenting of the left ICA performed as a first step could decrease the flow through the ECA, which in turn would

\section{Corresponding author:}

Paweł Latacz MD, PhD, Department of Vascular Surgery, University Hospital, 3 Botaniczna St, 31-503 Krakow, Poland, phone: +48 501 730 853, e-mail: pawlat@me.com

Received: 11.02.2015, accepted: 23.03.2015. 
compromise the cerebral circulation in the posterior part of the brain. Similarly, to prevent intraoperative stroke of the brainstem and cerebellum, surgical endarterectomy of the left ICA would require the use of an atypical shunt into the ECA, with potential technical problems associated with such a modified procedure. The first endovascular reconstruction was performed using femoral and radial artery vascular accesses. After cannulation of the femoral artery, a 6F 90-cm-long introducer sheath (Cook, Cook Medical Inc., Bloomington, IN, USA) was positioned in the aortic arch. We then navigated through the occluded left SA using Terumo Glidewire (Terumo, Tokyo, Japan) and V-18 ControlWire (Boston Scientific, Natick, MA, USA) hydrophilic-coated guidewires, and afterwards using 5F diagnostic catheters: VERT Beacon Tip Torcon NB Advantage Catheter (Cook Medical Inc, Bloomington, IN, USA) and Judkins Right (Cordis, Fremont, CA, USA). Once we had navigated through the occlusion, via the left radial access (5F sheath) we introduced a Hi-Torque coronary guidewire (Abbott Vascular, Abbott Park, IL, USA) into the left VA, and then via the femoral access we implanted a balloon-expandable $8 \times 59 \mathrm{~mm}$ Omnilink stent (Abbott Vascular, Abbott Park, IL, USA) into the left SA. Since the proximal portion of the left SA was patent, without atherosclerotic plaques, we did not implant the subclavian stent typically, i.e. with a protrusion of the stent into the aorta. In this particular case such a protrusion was not needed, and, moreover, a protruding stent could make future endovascular repair technically difficult. The expanded stent also covered the origin of the left VA, since atherosclerotic plaque occluding the left SA was also located in this area. Then, through the mesh wall of the subclavian stent over the 0.014-inch guidewire we introduced a $4.5 \times 20 \mathrm{~mm}$ Sterling Monorail angioplastic balloon (Boston Scientific, Natick, MA, USA) to the VA, and a $7 \times 59 \mathrm{~mm}$ angioplastic balloon (Omnilink, Abbott
Vascular, Abbott Park, IL, USA) to the stent. Afterwards we inflated both balloons, using the kissing-balloon technique, under the pressure of 10 bar. As a result, there was a good flow through the left SA and the left VA, with normalized circulation in the left upper extremity, as well as in the posterior part of the brain. Yet, there was still a reversed flow in the right VA (Figures $2 \mathrm{~A}-\mathrm{C}$ ).

The second stage of endovascular treatment, aimed primarily at the repair of the left ICA, was performed 40 days later. Control angiography confirmed the success of previous interventions, but still with the signs of right-sided subclavian steal syndrome and stenosis of the left ICA. We decided to reconstruct the lesions of the left ICA during a single procedure via the right femoral access. We introduced a $6 \mathrm{Fr} 90-\mathrm{cm}$-long introducer sheath (Cook, Cook Medical Inc., Bloomington, IN, USA) to the left common carotid artery and we performed angioplasty of the left ICA using a 3.5-5.5 mm FilterWire EZ distal protection system (Boston Scientific, Natick, MA, USA) and successfully implanted a $7 \times 30 \mathrm{~mm}$ self-expandable Carotid Wallstent stent (Boston Scientific, Natick, MA, USA) (Figures 2 D, E). There was good flow in both carotid and vertebral arteries after the procedure.

The patient was discharged after 4 days of hospitalization with the recommendation of dual antiplatelet therapy (aspirin $75 \mathrm{mg} /$ day and clopidogrel $75 \mathrm{mg}$ / day), antihypertensive therapy (ramipril $5 \mathrm{mg} /$ day and torasemide $2.5 \mathrm{mg} / \mathrm{day}$ ) and cholesterol-lowering treatment (atorvastatin $40 \mathrm{mg} /$ day). Control Doppler sonography performed 30 days later demonstrated good flow through implanted stents with no sonographic signs of stenosis.

Initially the reconstruction of the occluded BCT was planned as the third step, using a distal protection system, such as the Spider FX embolic protection device (ev3 Endovascular, Plymouth, MN, USA). Still, the timing
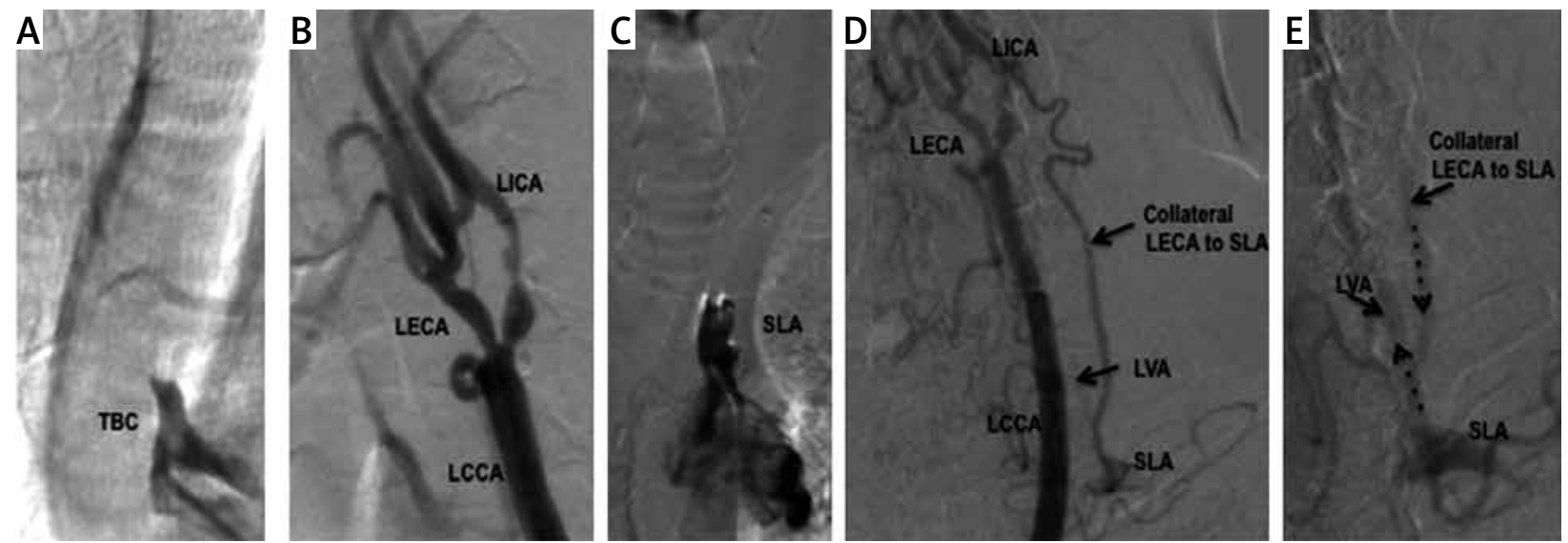

Figure 1. A - Occlusion of the brachiocephalic trunk (BCT), B - left subclavian artery (SLA) and C - 80-90\% stenosis of the left internal carotid artery (LICA). D, E - Atypical arterial inflow to the posterior part of the brain: from the left external carotid artery (LECA) through collaterals to the left subclavian artery (SLA), and then to the left vertebral artery (LVA), D - inflow through collaterals to the left subclavian artery, E - inflow to the proximal part of the left vertebral artery (arrows (---->) indicate direction of blood flow) 

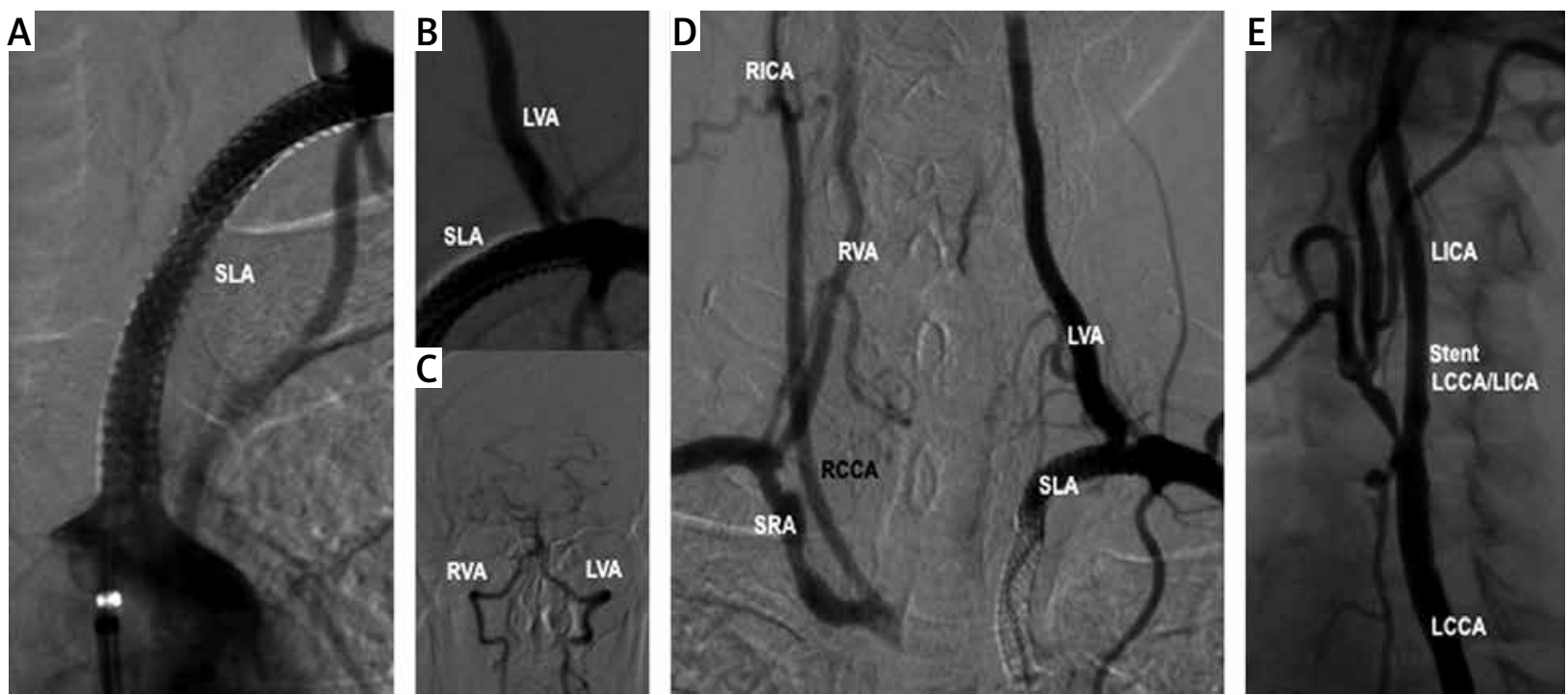

Figure 2. The result of angioplasty: A - with stenting of the left subclavian artery (SLA), B - with good inflow to the left vertebral artery (LVA) and C - backward flow in the right vertebral artery (RVA). D, E - Final result of endovascular treatment, with stent implantation to the left common and internal carotid artery (LCCA/LICA) angiography: D - SLA after the PTA + stent procedure and PTA LVA with visible circulation from the LVA to the RVA and back - to the right common carotid artery (RCCA) and internal (RICA), E - final results of angioplasty with implantation of stent in LICA

of such an endovascular repair of the BCT depended on the neurological status of the patient, primarily the existence of symptoms of brain ischemia. Since, for the time being, the patient is free of neurological symptoms, we decided to manage him conservatively.

\section{Discussion}

Arterial occlusive disease of the $\mathrm{BCT}$ is a rare cause of brain ischemia and is responsible for less than $2 \%$ of extracranial sources of brain ischemia [2]. Still, such a vascular lesion may result not only in retrograde blood flow in the right VA but also in flow reversal in both branches of the BCT - the VA and the common carotid artery - resulting in the so-called subclavian-carotid double steal syndrome [3-5]. There have also been described patients with simultaneous flow reversal in the VA and in the mammary artery coronary bypass (presenting as angina pectoris), which was caused by a proximal SA stenosis $[6,7]$, and also patients with subclavian steal syndrome associated with an additional "steal" of blood from the arm to the lungs through the patent arterial duct [8].

Here we have presented a unique double steal syndrome. We were able to find only a single description of a patient with a similar, though not identical, flow pattern. Still, this particular patient presented with vascular lesions caused by Takayasu disease, and not atherosclerosis [9]. In our patient, meticulous evaluation of cerebral hemodynamics and preprocedural planning of endovascular treatment of such a complex vascular pathology enabled the safe and successful treatment.

\section{Conflict of interest}

The authors declare no conflict of interest.

\section{References}

1. Contorni L. Il circelo colletrale vertebro-vertebrale nella obliterazione dell'arteria subclavian alla sua origine. Minerva Chir 1960; 15: 268.

2. Kapa S, Adams J. Subclavian steal and rest pain in a case of brachiocephalic artery occlusion. Int J Angiol 2008; 17: 166-7.

3. Willoughby AD, Kellicut DC, Ching BH, et al. Double steal syndrome: two case presentations. J Vasc Med Surg 2014; 2: 3.

4. Filis K, Toufektzian L, Sigala F, et al. Right subclavian double steal syndrome: a case report. J Med Case Rep 2008; 2: 392.

5. Maier S, Bajko Z, Motataianu A, et al. Subclavian double steal syndrome presenting with cognitive impairment and dizziness. Rom J Neurol 2014; 13: 144-9.

6. Samuels DA, Faitelson L. "Double steal" syndrome. J Interv Cardiol 1989; 2: 49-53.

7. Latacz P, Rostoff P, Rudnik A, et al. Percutaneous revascularisation of subacute occlusion of the left subclavian artery manifesting as acute coronary syndrome in a patient after coronary artery bypass grafting. Kardiol Pol 2010; 68: 226-9.

8. Koneti NR, Qureshi SA, Sivakumar K. Catheter interventions for "double steal" from isolation of the subclavian artery associated with patent arterial duct. Cardiol Young 2014; 24: 95-8.

9. Agee OF. Two unusual cases of subclavian steal syndrome bilateral steal and steal secondary to tumor thrombus. Am J Roentgenol 1966; 97: 447-57. 